\title{
LogDemons Revisited: Consistent Regularisation and Incompressibility Constraint for Soft Tissue Tracking in Medical Images
}

\author{
T. Mansi, X. Pennec, M. Sermesant, H. Delingette, and N. Ayache \\ INRIA-Méditerranée, AsclePIos Project, Sophia Antipolis, France
}

\begin{abstract}
Non-linear image registration is a standard approach to track soft tissues in medical images. By estimating spatial transformations between images, visible structures can be followed over time. For clinical applications the model of transformation must be consistent with the properties of the biological tissue, such as incompressibility. LogDemons is a fast non-linear registration algorithm that provides diffusion-like diffeomorphic transformations parameterised by stationary velocity fields. Yet, its use for tissue tracking has been limited because of the ad-hoc Gaussian regularisation that prevents implementing other transformation models. In this paper, we propose a mathematical formulation of demons regularisation that fits into LogDemons framework. This formulation enables to ensure volume-preserving deformations by minimising the energy functional directly under the linear divergence-free constraint, yielding little computational overhead. Tests on synthetic incompressible fields showed that our approach outperforms the original logDemons in terms of incompressible deformation recovery. The algorithm showed promising results on one patient for the automatic recovery of myocardium strain from cardiac anatomical and 3D tagged MRI.
\end{abstract}

\section{Introduction}

Tissue tracking in sequences of medical images is an important task in many applications, either for therapy guidance or diagnosis. However there is no easy way to achieve it, even interactively. A standard approach is now to use nonlinear image registration to estimate the spatial transformation between different images, for instance brain shift [7]. However, for such clinical applications oneto-one mapping must often be ensured and the model of transformations that is used must be consistent with the properties of the tissue to track. In particular, constraining the registration to be volume-preserving showed great improvement when tracking incompressible tissues such as the heart [3.9].

Mathematical frameworks based on diffeomorphic deformations 24] have been developed to get one-to-one mappings between the images to register. Among them, logDemons [14 is an efficient demons-based registration algorithm that provides diffeomorphic transformations parameterised by stationary velocity fields. However, although mathematical justifications of demons optimisation have been provided [14, theoretical foundations of the Gaussian regularisation

T. Jiang et al. (Eds.): MICCAI 2010, Part II, LNCS 6362, pp. 652 659, 2010.

(C) Springer-Verlag Berlin Heidelberg 2010 
still has to be consolidated [11], which makes the algorithm difficult to adapt to other deformation models.

Several approaches for incompressible image registration have been proposed. A first method is to constrain the Jacobian determinant of the transformations to equal 1 . However, this constraint is computationally demanding due to its nonlinearity [12. Linear approximations have been proposed [3] but volume drifts may appear at large deformations. Velocity fields can be made incompressible by constraining them to be divergence-free. Thus, incompressible fluid registration is achieved by projecting the update velocity onto the space of divergence-free vector fields 13 . Nevertheless, the fluid model might not be appropriate for tracking biological soft tissues like myocardium. In [9], we proposed an incompressible demons algorithm where the update velocity field was made divergencefree using Helmholtz decomposition. However, the approach was suboptimal as the constraint did not consider demons Gauss-Newton minimisation space. Furthermore, volume drifts were controlled using the linear approximation of the condition on deformations, which does not hold on large deformations.

This paper presents an efficient and consistent framework for demons-based incompressible registration. We first propose a mathematical justification of the Gaussian regularisation, which enables to integrate the incompressibility constraint seamlessly by working on the space of divergence-free velocity fields. The main advantages of this are: i) the constraint is linear with little computational overhead, ii) the parameter of the deformations are constrained: no volume drifts appear, iii) the transformation minimises a constrained energy functional: the optimal incompressibility field according to the logDemons minimisation scheme is found. The algorithm was validated against synthetic data and applied on clinical cardiac MRI to estimate 3D myocardium motion.

\section{Methods}

\subsection{Background: Log-Domain Diffeomorphic Demons}

LogDemons algorithm estimates a dense non-linear transformation $\phi$ that best aligns a template image $T$ to a reference image $R[14$. $\phi$ belongs to the space generated by the one-parameter subgroups of diffeomorphisms $\mathbb{G}$. They are parameterised by stationary velocity fields $\mathbf{v}$ through the exponential map $\phi=$ $\exp (\mathbf{v})$ [1]. The images $R$ and $T$ are registered by minimising in the space of velocities, called log-domain, the energy functional: $\mathcal{E}\left(\mathbf{v}, \mathbf{v}_{c}\right)=1 / \sigma_{i}^{2} \| R-T$ 。 $\exp \left(\mathbf{v}_{c}\right)\left\|_{L_{2}}^{2}+1 / \sigma_{x}^{2}\right\| \log \left(\exp (-\mathbf{v}) \circ \exp \left(\mathbf{v}_{c}\right)\right)\left\|_{L_{2}}^{2}+1 / \sigma_{d}^{2}\right\| \nabla \mathbf{v} \|^{2}$, where $\sigma_{i}^{2}$ relates to the noise in the images and $\sigma_{d}^{2}$ controls the regularisation strength. This equation is function of two variables: The velocity field $\mathbf{v}$ parameterises the transformation $\phi$ to recover whereas $\mathbf{v}_{c}$ parameterises an intermediate transformation $\phi_{c}=\exp \left(\mathbf{v}_{c}\right)$ that models the correspondences between the voxels of the two images. During the optimisation step, $\mathcal{E}\left(\mathbf{v}, \mathbf{v}_{c}\right)$ is minimised with respect to $\mathbf{v}_{c}$. This amounts to finding the optimal matching between $R$ and $T$ without considering the regularisation. Under the diffeomorphic update rule $\phi_{c} \leftarrow \phi \circ \exp (\delta \mathbf{v})$, the optimal update velocity writes $\delta \mathbf{v}(\mathbf{x})=-(R(\mathbf{x})-T \circ \phi(\mathbf{x})) /\left(\|J(\mathbf{x})\|^{2}+\sigma_{i}^{2} / \sigma_{x}^{2}\right) J(\mathbf{x})$. 
$J(\mathbf{x})$ is the symmetric gradient $J(\mathbf{x})=(\nabla R(\mathbf{x})+\nabla(T \circ \phi)(\mathbf{x})) / 2$. The correspondence velocity $\mathbf{v}_{c}$ is then updated using the first order approximation of BakerCampbell-Hausdorff $(\mathrm{BCH})$ formula $\mathbf{v}_{c}=Z(\mathbf{v}, \delta \mathbf{v})=\mathbf{v}+\delta \mathbf{v}+1 / 2[\mathbf{v}, \delta \mathbf{v}]+$ $1 / 12[\mathbf{v},[\mathbf{v}, \delta \mathbf{v}]]+O\left(\|\delta \mathbf{v}\|^{2}\right)$, where the Lie bracket $[\cdot, \cdot]$ is defined by $[\mathbf{v}, \delta \mathbf{v}]=$ $(\nabla \mathbf{v}) \delta \mathbf{v}-(\nabla \delta \mathbf{v}) \mathbf{v}$. This approach has experimentally shown promising results in terms of image registration and statistics on diffeomorphisms [414]. Finally, the regularisation step estimates the optimal regularised transformation $\phi$ by minimising $\mathcal{E}\left(\mathbf{v}, \mathbf{v}_{c}\right)$ with respect to $\mathbf{v}$, which is approximated by smoothing the correspondence velocity $\mathbf{v}_{c}$ with a Gaussian kernel $G_{\sigma}$. However, how $G_{\sigma}$ relates to $\mathcal{E}\left(\mathbf{v}, \mathbf{v}_{c}\right)$ remains to be consolidated [11.

\subsection{Revisiting Demons Gaussian Regularisation}

A consistent mathematical formulation of demons regularisation is required to integrate incompressibility in $\log$ Demons. In [10], the authors demonstrate that Gaussian filtering solves the Tikhonov estimation problem with equal weighting of the spatial derivatives in the Taylor series sense. We thus replace the $\log$ Demons regulariser $\|\nabla \mathbf{v}\|^{2}$ by the Tikhonov regulariser to get:

$$
\mathcal{E}_{\text {reg }}(\mathbf{v})=\frac{1}{\sigma_{x}^{2}}\left\|\log \left(\exp (-\mathbf{v}) \circ \exp \left(\mathbf{v}_{c}\right)\right)\right\|_{L_{2}}^{2}+\int_{\Omega} \sum_{k=1}^{+\infty}\left(\sum_{i_{1}+\ldots+i_{k}=k} \frac{\left\|\partial_{i_{1} . . i_{k}} \mathbf{v}\right\|^{2}}{\sigma_{x}^{2} \sigma_{d}^{2 k} k !}\right)
$$

In this equation, $\Omega$ is the image domain and $\partial_{i_{k} . . i_{l}}$ denotes the composition of spatial derivatives $\partial_{i_{k}} . . \partial_{i_{l}}$. The parameter $\sigma_{x}^{2}$ has been introduced into the regulariser to simplify calculations. More importantly, the regularisation weight $\sigma_{d}^{2}$ is now function of the derivative orders to preserve the shape of the impulse response related to the regulariser [10. The previous equation is minimised by linearising its first term using the zeroth order approximation of $\mathrm{BCH}$ formula, $\log \left(\phi^{-1} \circ \phi_{c}\right)=\mathbf{v}_{c}-\mathbf{v}$. The resulting equation, which is exactly a Tikhonov estimation problem, is minimised in the Fourier domain. The optimal velocity field $\mathbf{v}$ verifies $\sum_{k=0}^{\infty}\left(\mathbf{w}^{T} \mathbf{w}\right)^{k} /\left(\sigma_{d}^{2 k} k !\right) \hat{\mathbf{v}}(\mathbf{w})=\exp \left(\mathbf{w}^{T} \mathbf{w} / \sigma_{d}^{2}\right) \hat{\mathbf{v}}(\mathbf{w})=\hat{\mathbf{v}}_{c}(\mathbf{w})$, which is exactly demons Gaussian smoothing $G_{\sigma}$, with $\sigma^{2}=2 / \sigma_{d}^{2}$. The width of the Gaussian kernel corresponds to the strength of the regularisation.

This formulation is built up on two key elements. First, the correspondence field $\phi_{c}$ decouples the regularisation from the optimisation, making the minimisation of the registration energy independent of the optimisation. Second, the coupling term $\left\|\log \left(\phi^{-1} \circ \phi_{c}\right)\right\|^{2}$ is approximated as a least-square problem to get the Gaussian smoothing. These two elements must be ensured in any demons algorithm to justify the Gaussian regularisation.

\subsection{Incompressible LogDemons}

The proposed regulariser enables to integrate the incompressibility constraint into the algorithm. A transformation $\phi$ is locally incompressible if its Jacobian determinant $\operatorname{det}(\nabla \phi)=1$. In fluid dynamics, one uses the infinitesimal version: a fluid is said incompressible if the divergence of its velocity $\mathbf{v}$ is null. For 
diffeomorphic transformations one can show that the converse is true: integrating divergence-free velocities over time yields incompressible deformations [6]. Making LogDemons incompressible thus consists in constraining the velocity fields $\mathbf{v}$ to be divergence-free. Demons optimisation step is not modified, as it optimises $\mathbf{v}_{c}$ only, but demons regularisation energy is now optimised under the divergence-free constraint, which amounts to minimising the Lagrange function:

$$
\mathcal{P}(\mathbf{v}, p)=\frac{1}{\sigma_{x}^{2}}\left\|\mathbf{v}_{c}-\mathbf{v}\right\|_{L_{2}}^{2}+\sum_{k=1}^{+\infty}\left(\sum_{i_{1}+\ldots+i_{k}=k} \frac{\left\|\partial_{i_{1} . . i_{k}} \mathbf{v}\right\|^{2}}{\sigma_{x}^{2} \sigma_{d}^{2 k} k !}\right)-\frac{2}{\sigma_{x}^{2}} \int_{\Omega} p \nabla \cdot \mathbf{v}
$$

In this equation, the Lagrange multiplier $p$ is a scalar function of the Sobolev space $H_{0}^{1}(\Omega)$ that vanishes at infinity. Optima of (11) are found by solving $\partial_{\mathbf{v}} \mathcal{P}(\mathbf{v}, p)=0$ :

$$
\mathbf{v}+\sum_{k=1}^{\infty} \frac{(-1)^{k}}{\sigma_{d}^{2 k} k !} \Delta^{k} \mathbf{v}=\mathbf{v}_{c}-\nabla p
$$

with $p=0$ at the domain boundaries $\partial \Omega$. The divergence of (2) under the optimal condition $\nabla \cdot \mathbf{v}=0$ yields $\Delta p=\nabla \cdot \mathbf{v}_{c}$ with 0 -Dirichlet boundary conditions, which can be solved independently of $\mathbf{v}$ to get $p . \mathbf{g}=\mathbf{v}_{c}-\nabla p$ is thus the $L_{2}$ projection of $\mathbf{v}_{c}$ onto the space of divergence-free vector fields. From Sec. 2.2. we deduce the optimal incompressible velocity field: $\mathbf{v} \leftarrow G_{\sigma} \star \mathbf{g}$.

Particular care must be taken when incompressibility is required within a subdomain $\Gamma \subset \Omega$ only, for tracking localised incompressible organs like the heart. This is achieved by defining $p \in H_{0}^{1}(\Gamma), p=0$ on $\Omega / \Gamma$. Although Gaussian smoothing theoretically preserves the divergence, in practice unconstrained velocities close to $\Gamma$ boundaries $(\partial \Gamma)$ may leak inside the incompressible domain due to the Gaussian convolution, ultimately resulting in volume drifts. Yet, vector derivatives and well-designed Gaussian filters, like Deriche recursive filters, commute. We therefore replace the theoretical "project-and-smooth" strategy by a "smooth-and-project" approach that preserves divergence. To limit numerical instabilities, a smooth domain transition is implemented in a narrow band around $\Gamma$ by diffusing the pressure field $p$ using heat-transfer equation [6]. Algorithm 1 summarises the main steps of our method, henceforth termed iLogDemons, which was implemented using ITK [5] and PETSc.

\section{$3 \quad$ Experiments and Results}

\subsection{Experiments on Synthetic Datasets}

iLogDemons were tested on synthetic datasets with known ground truth. Eight $3 \mathrm{D}$ volume-preserving whirl transformations $\phi_{\alpha}$ were created with whirl angles $\alpha=10^{\circ}$ to $80^{\circ} 13$. Within the whirl domain, the $L_{2}$-norm varied from $0.52 \mathrm{~mm}$ to $4.78 \mathrm{~mm}$ while the Jacobian determinant stayed close to 1 (worse value $\left|\nabla \phi_{\alpha=80^{\circ}}\right|=1 \pm 0.04$ (mean \pm standard deviation SD)). A 3D isotropic Steady-State Free Precession (SSFP) MRI of the heart, cropped to focus on the heart, was warped with the $\phi_{\alpha}$ 's and altered with slight Gaussian noise (Fig. 1). 


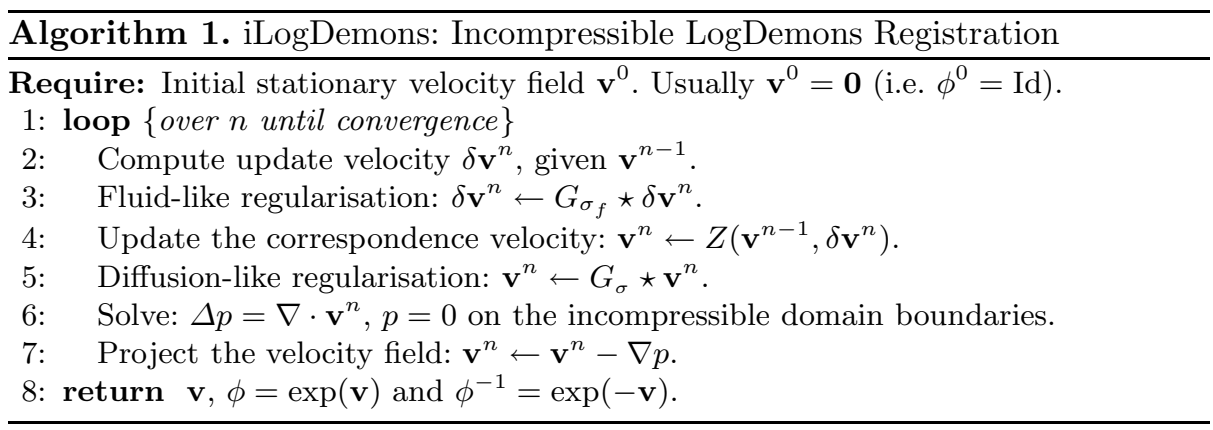

The 8 warped images $T$ were registered to the test image $R$ using LogDemons and iLogDemons $\left(\sigma_{x}=1 \mathrm{~mm}, \sigma^{2}=1 \mathrm{~mm}\right.$ and $\left.\sigma_{f}^{2}=1 \mathrm{~mm}\right)$. Relative mean square errors in grey level intensities $\left(R M S E=\|R-T \circ \phi\|_{L_{2}} /\|R-T\|_{L_{2}}\right)$, Jacobian determinant and distance to the true field $\phi_{\alpha}\left(D T F=\left\|\phi-\phi_{\alpha}\right\|_{L_{2}}\right)$ are reported in Fig. 1. Deformation fields estimated by iLogDemons were almost incompressible. Jacobian determinants were always equal to $1 \pm 0.02$ independently of the strength of the deformation to recover. Image matching accuracy was not affected by the incompressibility constraint (0.6\% decrease). But most importantly, iLogDemons significantly improved the accuracy of the deformations. Mean and SD of DTF were systematically lower (average improvements of $29 \%$ and $36 \%$ respectively). The larger the deformation, the more significant the improvement. This points out the importance of the choice of the deformation model. Regions with homogeneous grey levels provided little information to accurately estimate the underlying deformation (Fig. 1. yellow arrow). The incompressibility constraint coped with this limitation by ensuring that the estimated deformation was of the same type as the true field. Similar conclusions were drawn on experiments with other parameters $\left(\sigma_{x}=2, \sigma^{2}=2, \sigma_{f}^{2}=\{0.5,2\}\right)$.

\subsection{Application to Cardiac Deformation Recovery}

iLogDemons were then used to estimate the 3D left-ventricular myocardium strain from standard anatomical cine MRI of the heart. Such images have good in-plane and temporal resolutions but large slice thickness, which hampers the accurate estimation of cardiac through-plane motion (Fig. 2). As the volume of the heart is almost constant during the cardiac cycle, incompressible registration is believed to improve the estimation of the cardiac deformation.

Anatomical short axis cine SSFP MRI (cMRI) of a patient with heart failure were acquired with multiple breath-holds (Achieva, Philips Medical System, 30 frames, $1.5 \mathrm{~mm}^{2}$ isotropic in-plane resolution, $10 \mathrm{~mm}$ slice thickness). 3D tagged MRI (tMRI) were also acquired during the same exam (CSPAMM, 23 frames, $0.9 \mathrm{~mm}$ isotropic resolution, tag size $\approx 3 \mathrm{~mm}$ ). No manual tracking of the tag grids was available since this task is extremely difficult due to the $3 \mathrm{D}$ motion. All the images fully covered both ventricles and no slice misalignments were detected. The cMRI were linearly resampled to get isotropic voxel sizes. The tMRI were 

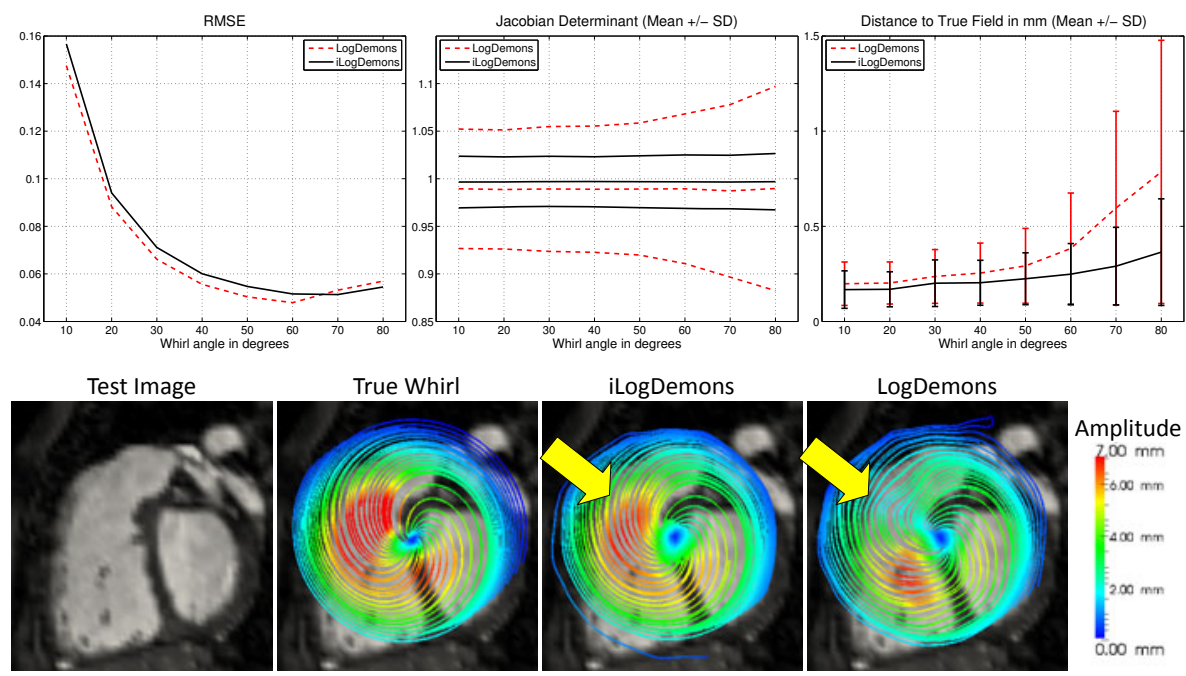

Fig. 1. Top row: Results on images warped by 3D synthetic volume-preserving whirls with increasing whirl angle. Bottom row: Streamlines of true and estimated whirl deformations (whirl angle $\alpha=60^{\circ}$ ). iLogDemons provided incompressible deformations and outperformed LogDemons in terms of deformation field accuracy (yellow arrow).

spatially and temporally aligned to the cMRI using DICOM information. Because the transformations provided by demons algorithm are resampling fields, myocardium deformation was estimated by recursively registering all the frames of the cardiac sequence to the end-diastole (ED) time frame, as in [9]. Registration parameters were $\sigma_{x}=1 \mathrm{~mm}, \sigma^{2}=2 \mathrm{~mm}, \sigma_{f}^{2}=0.5 \mathrm{~mm}$ (the smoothing was increased to accommodate the lower image quality). A 2-level multi-resolution scheme was used and registration was stopped as soon as RMSE stopped decreasing. Incompressibility constraint was applied only within the myocardium as volume of surrounding structures like blood pools vary.

First, we estimated the myocardium motion by tracking the heart in the 3D tMRI using iLogDemons. For visual assessment, the deformations were applied to virtual planes manually positioned at ED (Fig. 2, bottom panel). Realistic deformations consistent with the tag grids were obtained, which was further confirmed by the temporal variation of the radial, circumferiential and longitudinal myocardium strains (Fig. 2, green curve). These results were similar to those obtained with $\log$ Demons, as the tag grids provided enough texture information in the myocardium to guide the registration. Hence, as no ground truth was available, we considered the iLogDemons estimation as reference. We then estimated the $3 \mathrm{D}$ motion of the heart from the cMRI and compared the results with the reference tMRI motion (Fig. 2, blue and red curves). Visually, the warped virtual planes showed that incompressibility constraint did help to recover the longitudinal motion despite the large slice thickness of the cMRI. Estimated longitudinal and circumferential strains confirmed this finding. iLogDemons was closer to the reference than the $\log$ Demons (59\% of improvement for radial strain, $84 \%$ for 


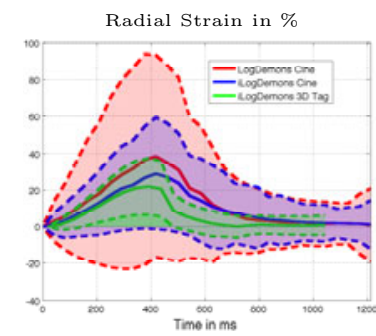

Circumferential Strain in \%
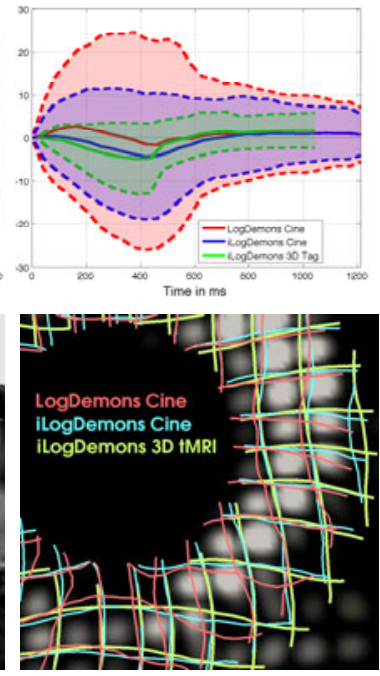

Short-Axis (In-Plane Motion)
Longitudinal Strain in \%
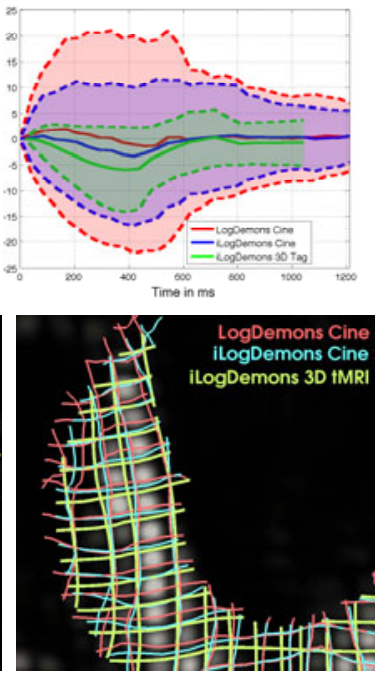

Long-Axis (Through-Plane Motion)

Fig. 2. Top panels: Myocardium strains computed from short-axis cine MRI and tMRI. Mean and standard deviation computed over the entire left ventricle. Bottom panels: Close-up of the tMRI at end-systole with warped tag planes overlaid. iLogDemons better estimates longitudinal and circumferential motion and strain.

circumferential strain and $42 \%$ for longitudinal strain). Radial strain amplitude was more reasonable and the variations of the circumferential and longitudinal strains presented realistic patterns [15], where logDemons estimated an abnormal lengthening at the beginning of the cardiac contraction.

\section{Discussion and Future Works}

We have adapted $\log$ Demons algorithm to provide incompressible deformations. This has been possible by showing that demons Gaussian smoothing minimises an infinite order Tikhonov regulariser. This framework opens the way to new regularisers, such as elastic regularisation. As a result, incompressibility could be ensured by constraining the velocities to be divergence-free. The proposed incompressibility constraint does not introduce any new parameter. Those listed in this paper are present in any recent demons algorithm [14. One could constrain the correspondence velocity to find the optimal incompressible update deformation. Yet, non-reported experiments showed that this does not significantly improve the results compared to iLogDemons: The updates are usually small and thus near-incompressible. The next step is to modify the demons energy to automatically handle incompressibility in subdomains of the image. From a clinical point of view, we are currently validating this method for the automatic estimation of 3D myocardium strain from standard cardiac images. 
Acknowledgements. This work has been partly funded by the European Commission, IST-2004-027749 Health-e-Child Integrated Project. The authors warmly thank the King's College London, St Thomas Hospital, for the cine and tagged MRI.

\section{References}

1. Arsigny, V., Commowick, O., Pennec, X., Ayache, N.: A log-euclidean framework for statistics on diffeomorphisms. In: Larsen, R., Nielsen, M., Sporring, J. (eds.) MICCAI 2006. LNCS, vol. 4190, p. 924. Springer, Heidelberg (2006)

2. Beg, M., Miller, M., Trouvé, A., Younes, L.: Computing Large Deformation Metric Mappings via Geodesic Flows of Diffeomorphisms. IJCV 61(2), 139-157 (2005)

3. Bistoquet, A., Oshinski, J., Skrinjar, O.: Myocardial deformation recovery from cine MRI using a nearly incompressible biventricular model. Medical Image Analysis 12(1), 69-85 (2008)

4. Bossa, M., Hernandez, M., Olmos, S.: Contributions to 3D diffeomorphic atlas estimation: application to brain images. In: Ayache, N., Ourselin, S., Maeder, A. (eds.) MICCAI 2007, Part I. LNCS, vol. 4791, pp. 667-674. Springer, Heidelberg (2007)

5. Dru, F., Vercauteren, T.: An ITK implementation of the symmetric log-domain diffeomorphic demons algorithm. Insight Journal (May 2009)

6. Evans, L.C.: Partial Differential Equations (June 1998)

7. Ferrant, M., Nabavi, A., Macq, B., Jolesz, F., Kikinis, R., Warfield, S.: Registration of 3D intraoperative MR images of the brain using a finite element biomechanical model. IEEE TMI 20(12), 1384-1397 (2001)

8. Hinkle, J., Fletcher, P., Wang, B., Salter, B., Joshi, S.: 4D map image reconstruction incorporating organ motion. In: Karssemeijer, N., Lelieveldt, B. (eds.) IPMI 2007. LNCS, vol. 4584, p. 687. Springer, Heidelberg (2007)

9. Mansi, T., Peyrat, J.M., Sermesant, M., Delingette, H., Blanc, J., Boudjemline, Y., Ayache, N.: Physically-constrained diffeomorphic demons for the estimation of 3D myocardium strain from cine-MRI. In: Ayache, N., Delingette, H., Sermesant, M. (eds.) FIMH 2009. LNCS, vol. 5528, pp. 201-210. Springer, Heidelberg (2009)

10. Nielsen, M., Florack, L., Deriche, R.: Regularization, scale-space, and edge detection filters. In: JMIV, pp. 291-307. Springer, Heidelberg (1997)

11. Pennec, X., Cachier, P., Ayache, N.: Understanding the "demon's algorithm": 3D non-rigid registration by gradient descent. In: Taylor, C., Colchester, A. (eds.) MICCAI 1999. LNCS, vol. 1679, pp. 597-605. Springer, Heidelberg (1999)

12. Rohlfing, T., Maurer Jr., C., Bluemke, D., Jacobs, M.: Volume-preserving nonrigid registration of MR breast images using free-form deformation with an incompressibility constraint. IEEE TMI 22(6), 730-741 (2003)

13. Saddi, K.A., Chefd'hotel, C., Cheriet, F.: Large deformation registration of contrast-enhanced images with volume-preserving constraint. In: SPIE Medical Imaging, vol. 6512 (2007)

14. Vercauteren, T., Pennec, X., Perchant, A., Ayache, N.: Symmetric log-domain diffeomorphic registration: A demons-based approach. In: Metaxas, D., Axel, L., Fichtinger, G., Székely, G. (eds.) MICCAI 2008, Part I. LNCS, vol. 5241, pp. $754-$ 761. Springer, Heidelberg (2008)

15. Moore, C., Lugo-Olivieri, C., McVeigh, E., Zerhouni, E.: Three-dimensional systolic strain patterns in the normal human left ventricle: Characterization with tagged MR imaging. Radiology 214(2), 453-466 (2000) 